\title{
Automated assembly of a reference taxonomy for phylogenetic data synthesis
}

\author{
Jonathan A. Rees ${ }^{\ddagger}$, Karen Cranston \\ ‡ Duke University, Durham, United States of America
}

Corresponding author: Jonathan A. Rees (jar581@mumble.net)

Academic editor: Jeremy Miller

Received: 07 Mar 2017| Accepted: 12 May 2017| Published: 22 May 2017

Citation: Rees J, Cranston K (2017) Automated assembly of a reference taxonomy for phylogenetic data

synthesis. Biodiversity Data Journal 5: e12581. https://doi.org/10.3897/BDJ.5.e12581

\begin{abstract}
Taxonomy and nomenclature data are critical for any project that synthesizes biodiversity data, as most biodiversity data sets use taxonomic names to identify taxa. Open Tree of Life is one such project, synthesizing sets of published phylogenetic trees into comprehensive summary trees. No single published taxonomy met the taxonomic and nomenclatural needs of the project. Here we describe a system for reproducibly combining several source taxonomies into a synthetic taxonomy, and we discuss the challenges of taxonomic and nomenclatural synthesis for downstream biodiversity projects.
\end{abstract}

\section{Keywords}

taxonomy phylogeny automation pipeline synthesis

\section{Introduction}

Any large biodiversity data project requires one or more taxonomies for discovery and data integration purposes, as in "find occurrence records for primates" or "find the taxon record associated with this sequence" (Page 2008). Examples of such projects are GBIF 
(Edwards 2004), which focuses on occurrence records, and NCBI (Federhen 2011), which focuses on genetic sequence records. Each of these projects has a dedicated taxonomy effort that is responsive to the project's particular needs. We present the design and application of the Open Tree Taxonomy, which serves the Open Tree of Life project, an aggregation of phylogenetic trees with tools for operating on them. (Hinchliff et al. 2015McTavish et al. 2015, Redelings and Holder 2017, Open Tree of Life project 2017). In order to meet Open Tree's project requirements, the taxonomy is an automated assembly of ten different source taxonomies. The assembly process is repeatable so that we can easily incorporate updates to source taxonomies. Repeatability also allows us to easily test potential improvements to the assembly method. Information about taxa is typically expressed in databases and files in terms of taxon names or 'name-strings' (Patterson 2014). To combine taxonomies it is therefore necessary to be able to determine name equivalence: whether or not an occurrence of a name-string in one data source refers to the same taxon as a given name-string occurrence in another. Solving this equivalence problem requires that we distinguishing occurrences that only coincidentally have the same name-string (homonym sense detection), and unify occurrences only when evidence justifies it. We have developed a set of heuristics that scalably address this equivalence problem.

\section{The Open Tree of Life project}

The Open Tree of Life project consists of a set of tools for:

- $\quad$ synthesizing summary phylogenetic trees ('synthetic trees') from a corpus of phylogenetic tree inputs (input trees)

- $\quad$ matching groupings in synthetic trees with higher taxa (such as Mammalia)

- $\quad$ supplementing synthetic trees with taxa obtained only from taxonomy.

The outcome is one or more synthetic trees combining phylogenetic and taxonomic knowledge. Fig. 1 illustrates an overview of the process of combining phylogenetic trees and taxonomy, while the details are described in a separate publication (Redelings and Holder 2017).

Although Open Tree is primarily a phylogenetic tree aggregation effort, it requires a reference taxonomy that can support each of these functions.

For synthetic tree synthesis (1), we use the taxonomy for converting OTUs (operational taxonomic units, or 'tips') on input trees to a canonical form. Synthetic tree construction requires that an input tree OTU be matched with an OTU from another input tree when, and only when, it is reasonable to do so. This is a nontrivial task because a taxon can have very different OTU labels in different input trees due to synonymies, abbreviations, misspellings, notational differences, and so on. In addition, a given label can name different taxa in different trees (homonymy). The approach we take is to map OTUs to the reference taxonomy, so that OTUs in different input trees are compared by comparing the taxa to which they map. 


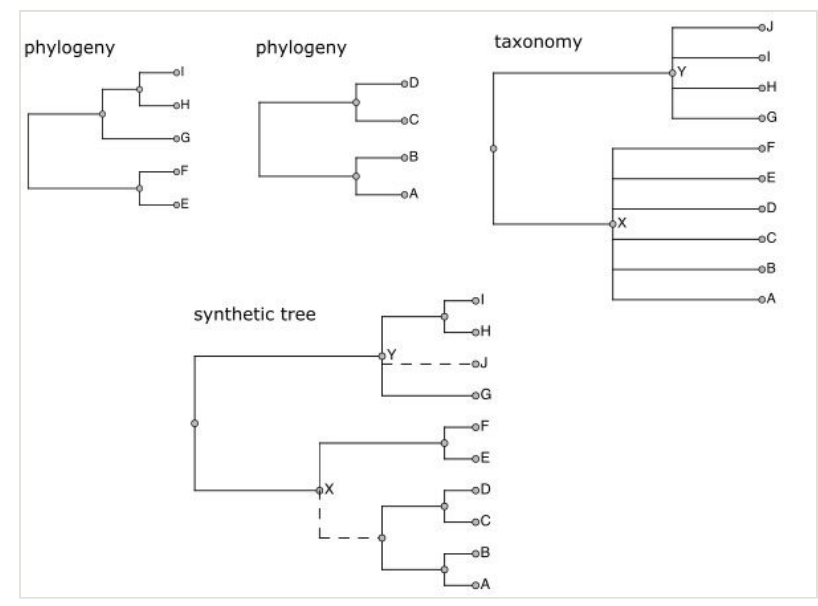

Figure 1. doi

Role of taxonomy in assembly of the Open Tree of Life synthetic phylogenetic tree. Dotted lines in the synthetic tree are those that come only from taxonomy, while solid lines have phylogenetic support. The taxonomy both links disjoint phylogenies and adds taxa not present in input trees.

For higher taxon associations (2), we compare the groupings in the synthetic tree to those in the taxonomy.

For supplementation (3), only a relatively small number of described taxa are represented in input trees (currently about 200,000 in the phylogenetic corpus out of two million or more known taxa), so the taxonomy provides those that are not. The large complement of taxonomy-only taxa can be 'grafted' onto a synthetic tree in phylogenetically plausible locations based on how they relate taxonomically to taxa that are known from input trees.

\section{Reference taxonomy requirements}

This overall program dictates what we should be looking for in a reference taxonomy. In addition to the technical requirements derived from the above, we have two additional requirements coming from a desire to situate Open Tree as ongoing infrastructure for the evolutionary biology community, rather than as a one-off study. Following are all five requirements:

1. OTU coverage: The reference taxonomy should have a taxon at the level of species or higher for every OTU that has the potential to occur in more than one study, over the intended scope of all cellular organisms.

2. Phylogenetically informed classification: Higher taxa should be provided with as much resolution and phylogenetic fidelity as is reasonable. Ranks and nomenclatural structure should not be required (since many well-established groups do not have proper Linnaean names or ranks) and groups at odds with phylogenetic understanding (such as Protozoa) should be avoided. 
3. Taxonomic coverage: The taxonomy should cover as many as possible of the species that are described in the literature, so that we can supplement synthetic trees as described in step 3 above.

4. Ongoing update: New taxa of importance to phylogenetic studies are constantly being added to the literature. The taxonomy needs to be updated with new information on an ongoing basis.

5. Open data: The taxonomy must be available to anyone for unrestricted use. Users should not have to ask permission to copy and use the taxonomy, nor should they be bound by terms of use that interfere with further reuse.

An additional goal is that the process should be reproducible and transparent. Given the source taxonomies, we should be able to regenerate the taxonomy, and taxon records should provide information about the taxonomic sources from which it is derived.

No single available taxonomic source meets all requirements. The NCBI taxonomy has good coverage of OTUs, provides a rich source of phyogenetically informed higher taxa, and is open, but its taxonomic coverage is limited to taxa that have sequence data in GenBank (only about 360,000 NCBI species having standard binomial names at the time of this writing). Traditional all-life taxonomies such as Catalogue of Life, IRMNG (Rees 2008), and GBIF meet the taxonomic coverage requirement, but miss many OTUs from our input trees, and their higher-level taxonomies are often not as phylogenetically informed or resolved as the NCBI taxonomy. At the very least, Open Tree needs to combine an NCBIlike sequence-aware taxonomy with a traditional broad taxonomy that is also open.

These requirements cannot be met in an absolute sense; each is a 'best effort' requirement subject to availability of project resources.

Note that the Open Tree Taxonomy is not supposed to be a reference for nomenclature; it links to other sources for nomenclatural and other information. Nor is it a place to deposit curated taxonomic information. The taxonomy has not been vetted in detail, as doing so would be beyond the capacity and focus of the Open Tree project. It is known to contain many taxon duplications and technical artifacts. Tolerating these shortcomings is a necessary tradeoff in attempting to meet the above requirements.

\section{Related work}

There are probably about a dozen public all-life taxonomy compilations. The methods of assembly and curation are documented for only a few of these. We assume that most are evolving databases that are extended and maintained by a combination of single-record operations and some amount of ad hoc scripting to import material in bulk. The NCBI taxonomy, described in Federhen 2011, is of this type. Catalogue of Life, which documents its method in Species 2000 2017, is different in that it has a divide and conquer approach: it is assembled through systematic grafting of sub-taxonomies received from a network of editors. Because its sub-taxonomies are nonoverlapping, CoL is not a synthesis in the sense used here. The GBIF backbone taxonomy is assembled via automated synthesis of overlapping sub-taxonomies, and in that respect is similar to OTT. The GBIF method is as 
yet unpublished, although some information is available (Döring 2016b, Döring 2016a). Note that OTT builds on GBIF, which in turn builds on CoL.

Had an available all-life taxonomy met all of Open Tree's requirements, the project would have used it. Unfortunately, for each one, there is at least one Open Tree requirement that goes beyond what the taxonomy provides.

An important part of any synthesis method is name-string parsing and matching, and this is the focus of the Global Names Architecture (Pyle 2016). In the phase of work reported here, name parsing and matching are not a priority issue, since exact matches together with synonym records from the source taxonomies provide a provisional solution that has been adequate so far. Going forward, matching will need more attention, and components of the GNA will probably play a role.

The Taxonomy Tree Tool (TTT, Lin et al. 2016) employs a tree merge method similar to the one used here. It seems to be aimed at assisting manual analysis of tree differences. It is not clear from available documentation how one would use it to resolve conflicts in an automated workflow.

\section{Method}

The conventional approach to meeting the requirements stated in the introduction would be to create a database, copy the first taxonomy into it, then somehow merge the second taxonomy into that, repeating for further sources if necessary. However, it is not clear how to meet the ongoing update requirement under this approach. As the source taxonomies change, we would like for the combined taxonomy to contain only information derived from the latest versions of the sources, without residual information from previous versions. Many changes to the sources are corrections, and we do not want to retain information that has been corrected or superseded by a later version of a source.

Rather than maintain a database of taxonomic information, we instead developed a process for assembling a taxonomy from two or more taxonomic sources. With a repeatable process, we can generate a new combined taxonomy version from new source taxonomy versions de novo, and do so frequently. There are additional benefits as well, such as the ability to add new sources relatively easily, and to use the tool for other purposes.

In the following, any definite claims or measurements refer to the Open Tree reference taxonomy version 3.0.

\section{Terminology}

- $\quad$ source taxonomy: imported taxonomic source (NCBI taxonomy, etc.)

- workspace: data structure for creation of the reference taxonomy 
- name-string: name of one or more taxa, without author information, considered as a sequence of characters, without association with any particular description, or nomenclatural code

- node: a data record intended to correspond to a taxon. Records name-strings, authorship, parent node, optional rank, optional annotations. If a workspace node, it originates from a single source taxonomy, and records its source (provenance) and its alignments to others

- $\quad$ parent (node): the nearest enclosing node within a given node's taxonomy

- tip: a node that is not the parent of any node

- primary name-string: one particular name-string of a node. Each node has exactly one primary name-string

- homonym name-string: a name-string that belongs to multiple nodes within the same taxonomy. This is analogous to the nontechnical meaning of 'homonym' and is not to be confused with 'homonym' in the nomenclatural sense, which only applies within a single nomenclatural code. Nomenclatural homonyms and hemihomonyms (Shipunov 2011) both correspond to homonym name-strings, as do clerical errors where multiple nodes are created for the same taxon

- $\quad$ synonym name-string (of a node): a non-primary name-string

- $\quad$ image (of a node $n^{\prime}$ ): the workspace node corresponding to $n^{\prime}$

- incertae sedis: node $A$ is incertae sedis in node $B$ if $A$ is a child of $B$ but is not known to be disjoint (as a taxon) from B's non-incertae-sedis children. That is, if we had more information, it might turn out that $A$ is a member of one of the other children of $B$.

\section{Method overview}

This section is an overview of the taxonomy assembly method. Several generalities stated here are simplifications; the actual method (described later) is significantly more involved.

We start with a sequence of source taxonomies $\mathrm{S} 1, \mathrm{~S} 2, \ldots, \mathrm{Sn}$, ordered by priority. Priority is the means by which conflicts between sources are resolved, and therefore has a profound effect on the outcome of assembly. If a curator judges $S$ to be more accurate or otherwise "better" than $S$ ', then $S$ will occur earlier in the priority sequence than $S$ ' and its information supersedes that from later sources. Curators (either project personnel or participants in Open Tree workshops and online forums) determine priority based on their taxonomic expertise. Source taxonomies are sometimes split into pieces in order to establish different priorities for different parts. Priority choice by curator is a fragile and subjective aspect of the method, but we could not identify any other information available at scale that could be brought to bear on conflict resolution.

We define an operator for combining taxonomies pairwise, written schematically as $U=S+$ $S^{\prime}$, and apply it from left to right:

$\mathrm{U} 0=$ empty, $\mathrm{U} 1=\mathrm{U} 0+\mathrm{S} 1, \mathrm{U} 2=\mathrm{U} 1+\mathrm{S} 2, \mathrm{U} 3=\mathrm{U} 2+\mathrm{S} 3 \ldots$ 
The combination $S+S^{\prime}$ is formed in two steps:

1. A mapping or alignment step that identifies all nodes in $S^{\prime}$ that can be equated with nodes in $S$. There will often be nodes in $S^{\prime}$ that cannot be aligned to $S$.

2. A merge step that creates the combination $U=S+S^{\prime}$, by adding to $S$ the unaligned taxa from $S^{\prime}$. The attachment position of unaligned nodes from step 1 is determined from nearby aligned nodes, either as a graft or an insertion.

Examples of these two cases are given in Figure 2.

As a simple example, consider a genus represented in both taxonomies, but containing different species in the two:

$S=(b, c, d) a, S^{\prime}=(c, d, e) a$

S and $S^{\prime}$ each have four nodes. Suppose $\mathrm{c}, \mathrm{d}$, and a in $\mathrm{S}^{\prime}$ are aligned to $\mathrm{c}, \mathrm{d}$, and a in $\mathrm{S}$. The only unaligned node is $e$, which is a sibling of $c$ and $d$ and therefore grafted as a child of a. After the merge step, we have:

$\mathrm{S}+\mathrm{S}^{\prime}=(\mathrm{b}, \mathrm{c}, \mathrm{d}, \mathrm{e}) \mathrm{a}$

One might call this merge heuristic 'my sibling's sibling is my sibling' or 'transitivity of siblinghood'.

This is a very common pattern. Fig. 2 illustrates a real life-example when combining the genus Bufo across NCBI and GBIF. There are about 900,000 similar simple grafting events in the assembly of OTT.

The other merge method is an insertion, where the unaligned node has descendants that are in S. This always occurs when S' has greater resolution than S. For example, see Fig. 2, where WoRMS provides greater resolution than NCBI.

The vast majority of alignment and merge situations are simple, similar to the examples shown in Fig. 2. However, even a small fraction of special cases can add up to thousands when the total number of alignments and merges measures in the millions, so we have worked to develop heuristics that handle the most common special cases. Ambiguities caused by homonym name-strings create most of the difficulties, with inconsistent or unclear higher taxon membership creating the rest. The development of the assembly process described here has been a driven by trial and error - finding cases that fail and then adding or modifying alignment heuristics and other logic to address the underlying cause. Because the sources are noisy and inconsistent, any automated assembly process will make mistakes. To prevent or correct these mistakes, manual ad hoc adjustments are applied as needed, as a last resort. The goal in method development is to keep the number of needed adjustments small. 
A. Grafting

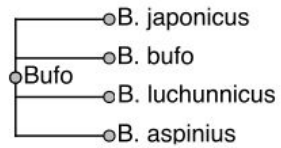

NCBI

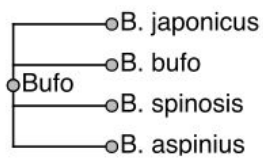

GBIF

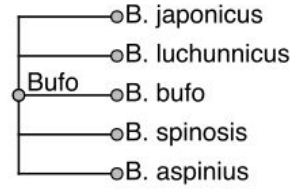

OTT

B. Insertion

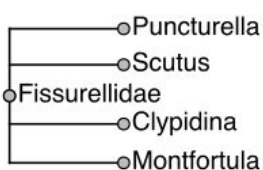

NCBI

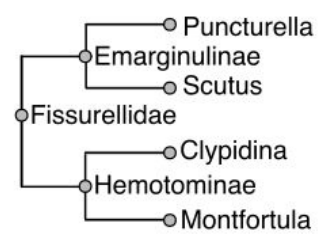

WORMS

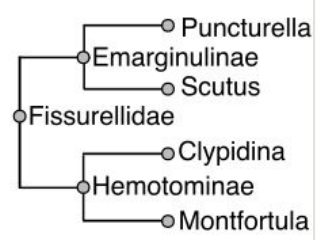

OTT

Figure 2. doi

Examples of grafting and insertion when combining taxonomies. In both cases, the $\mathrm{NCBI}$ taxonomy has higher priority than GBIF. In A (grafting), we assemble the genus Bufo across $\mathrm{NCBI}$ and GBIF. There is no $B$. spinosis in GBIF and no B. luchunnicus in NCBI. Therefore, the Bufo in the combined taxonomy has as its children copies of species records from both sources. In B (insertion), WoRMS provides greater resolution of Fissurellidae than NCBI taxonomy: it divides the family into subfamilies Hemotominae and Emarginulinae, nodes that do not exist in NCBI. The subfamilies are 'inserted' in a way that adds information without disrupting existing relationships from NCBI.

\section{Taxonomic sources}

We build the taxonomy from ten sources. Some of these sources are from taxonomy projects, while others were manually assembled based on recent publications. As described above, OTT assembly is dependent on the input order of the sources - higher ranked inputs take priority over lower ranked inputs. Table 1 lists the sources used to construct OTT. The full provenance details, and a copy of the normalized source, are available in supplementary data.

Open Tree curation : It is not uncommon to have taxa as OTUs in phylogenetic studies that do not occur in OTT. This can be due to a delay in curation by the source taxonomy, a delay in importing a fresh source version into OTT, a morphological study containing otherwise unknown species, or other causes. To handle this situation, we developed a user interface that allows curators to create new taxon records along with relevant documentation (publications, databases, and so on). New taxon records are saved into a public GitHub repository, and these records are then linked from the OTT taxonomy files and user interfaces so that provenance is always available.

Separation taxa : This is a small curated tree containing 31 major groups such as Fungi, Metazoa, and Lepidoptera. Its purpose is to assist in alignment, where homonym namestrings are, or might need to be, present. If a node is found in one of these separation 
groups, then it will not match a node in a disjoint separation group, absent other evidence (details below).

Table 1.

List of taxonomic sources: The ten sources used in v3.0 of Open Tree Taxonomy. Six are online taxonomic resources (SILVA (Quast et al. 2013), Index Fungorum (Index Fungorum Partnership 2014), WoRMS (WoRMS Editorial Board 2015), NCBI, GBIF, IRMNG), two are from publications (Hibbett et al. 2007, Schäferhoff et al. 2010), one is a small curated taxonomy of major groups to aid in assembly ("separation taxa"), and one consisting of taxonomic additions from phylogenies input into the Open Tree system ("Open Tree curation"). See text for explanation of 'Open Tree curation' and 'separation taxa'. Detailed provenance information for each source can be found in the accompanying data package. 'Focus' refers to the taxa of interest to Open Tree curators motivating inclusion in assembly. Key to 'reasons' column: $\mathrm{O}=$ added in order to improve OTU coverage; $\mathrm{P}=$ added in order to improve phylogenetic classification; $\mathrm{T}=$ added in order to improve taxonomic coverage.

\begin{tabular}{|l|l|l|l|l|l|}
\hline Name & OTT Focus & Taxa & Synonyms & Priority order & Reasons \\
\hline separation taxa & life & 31 & 8 & 1 & \\
\hline SILVA & life & 78687 & 0 & 2 & P \\
\hline Hibbett 2007 & Fungi & 227 & 0 & 3 & P \\
\hline Index Fungorum & Fungi & 284973 & 157734 & 4 & P,T \\
\hline Schäferhoff 2010 & Lamiales & 119 & 0 & 5 & P \\
\hline WoRMS & Malacostraca, Cnidaria & 330412 & 223196 & 6 & P,T \\
\hline NCBI & life & 1488029 & 719526 & 7 & O,P,T \\
\hline GBIF & life & 3273321 & 1143026 & 8 & T \\
\hline IRMNG & life & 1706655 & 685983 & 9 & T \\
\hline Open Tree curation & n/a & $n / a$ & $n / a$ & 10 & O \\
\hline
\end{tabular}

ARB-SILVA taxonomy processing: The terminal taxa in the SILVA taxonomy are algorithmically generated clusters of RNA sequences derived from GenBank records. Rather than incorporate these idiosyncratic, fine-grained groupings into OTT, we use sequence record metadata to place the clusters into larger groups corresponding to NCBI taxa, and include those larger groups in OTT.

We excluded SILVA's plant, animal, and fungal branches from OTT because these groups are well covered by other sources and poorly represented in SILVA. For example, SILVA has only 299 taxa in Metazoa, compared with over 500,000 taxa under Metazoa in NCBI Taxonomy.

Extinct / extant annotations : Curators requested information about whether taxa were extinct vs. extant. With the exception of limited data from WoRMS and Index Fungorum, 
this information was not explicitly present in our other sources, so we imported IRMNG, which logs the extinct / extant status of taxa.

As a secondary heuristic, records from GBIF that originate from PaleoDB, and do not come from any other taxonomic source, are annotated extinct. This is not completely reliable, as some PaleoDB taxa are extant.

Suppressed records : We suppress the following source taxonomy records:

- $\quad$ animals, plants, fungi in SILVA

- $\quad$ GBIF backbone records that originate from IRMNG (IRMNG is imported separately)

- $\quad$ GBIF backbone records that originate from IPNI

- $\quad$ GBIF backbone records whose taxonomic status is 'doubtful'

- $\quad$ GBIF backbone records for infraspecific taxa (subspecies, variety, form)

- IRMNG records whose nomenclatural status is 'nudum', 'invalid', or any of about 25 similar designations

- NCBI Taxonomy records that have no potential for unification with OTUs in phylogenetic studies: those with name-strings containing 'insertion sequences', 'artificial librarries', 'transposons', or any of about 15 similar designations

The IPNI and IRMNG derived GBIF records are suppressed because they include many invalid names. We pick up most of the valid names from other sources, such as the direct IRMNG import, so this is not a great loss. Although GBIF's original taxonomic sources indicate which names are known to be invalid, this information is not provided by the GBIF backbone. Note that the GBIF backbone might import the same name from more than one source, but its provenance information only lists one of the sources. We suppress the record if that one source is IPNI or IRMNG.

Sources not included: The number of sources was of course limited by the amount of time we had available for import efforts; new sources were only added for specific reasons related to curators' interests. The choice of sources was also limited by the open data goal. Certain obvious choices, such as Catalog of Life, had to be passed over because there was no access, or access was controlled by legal terms of use.

\section{Import and Normalization}

Each source taxonomy has its own import procedure, usually a file download from the provider's web site followed by application of a script that converts the source to a common internal form for import (a set of nodes, see terminology section). Given the converted source files, the taxonomy can be read by the OTT assembly procedure.

After each source taxonomy is loaded, the following normalizations are performed:

1. Diacritics removal - accents, umlauts, and other diacritic marks are removed in order to improve name matching, as well as to follow the nomenclatural codes, which prohibit them. The original name-string is kept. 
2. Child taxa of "containers" in the source taxonomy are made to be children of the container's parent. "Containers" are groupings in the source that don't represent taxa, for example nodes named "incertae sedis" or "environmental samples". The members of a container aren't more closely related to one another than they are to the container's siblings; the container is only present as a way to say something about the members. The fact that a node had originally been in a container is recorded as a flag on the child node.

3. When a subgenus $X$ has the same name-string as its containing genus, its namestring is changed to "X subgenus $X "$. This follows a convention used by NCBI Taxonomy and helps distinguish the two taxa later in assembly.

4. Sibling taxa with the same name-string are combined.

The normalized versions of the taxonomies then become the input to subsequent processing phases.

\section{Aligning nodes across taxonomies}

This section and the next give details of the taxonomy combination method introduced above.

OTT is assembled in a temporary work area or workspace by alternately aligning a source to the workspace and merging that source into the workspace. It is important that source taxonomy nodes be matched with workspace nodes when and only when this is appropriate. A mistaken identity between a source node and a workspace node can be disastrous, leading not just to an incorrect classification but to downstream curation errors in OTU matching (e.g. putting a snail in flatworms). A mistaken non-identity (separation) can also be a problem, since taxon duplication (i.e. multiple nodes for the same taxon) leads to loss of unification opportunities in tree synthesis.

As described above, source taxonomies are processed (aligned and merged) in priority order. For each source taxonomy, ad hoc adjustments are applied before automatic alignments. For automatic alignment, alignments closest to the tips of the source taxonomy are found in a first pass, and all others in a second pass. The two-pass structure permits first-pass alignments to be used during the second pass (see Overlap, below).

\section{Ad hoc adjustments}

A set of ad hoc 'adjustments' address known issues that are beyond the capabilities of the automated process to address. These often reflect either errors or missing information in source taxonomies, discovered through the failure of automated alignment, and confirmed manually via the literature. Although each individual adjustment is ad hoc, i.e. not the result of automation, the adjustments are recorded in a file that can be run as a script. Following are some examples of adjustments.

1. capitalization and spelling repairs (e.g. change 'sordariomyceta' to 'Sordariomyceta') 
2. addition of synonym name-strings to facilitate later matching (e.g. 'Protaspis' added for 'Protaspa')

3. selecting a different name-string as primary (e.g. 'Choanomonada' to 'Choanoflagellida')

4. deletions (e.g. removing synonym 'Eucarya' for 'Eukaryota' to avoid confusing eukaryotes with genus Eucarya in Magnoliopsida; or removing unaccepted genus Tipuloidea in Hemiptera to avoid confusion with the superfamily in Diptera)

5. merges to repair redundancies in the source (e.g. Pinidae, Coniferophyta, Coniferopsida)

6. rename nodes to avoid confusing homonym name-strings (e.g. there are two Cyanobacterias in SILVA, one a parent of the other; the parent is renamed to its NCBI name 'Cyanobacteria/Melainabacteria group')

7. alignments when names differs (Diatomea is Bacillariophyta)

8. alignments to override automated alignment rules (Eccrinales not in Fungi, Myzostomatida not in Annelida)

In the process of assembling the reference taxonomy, about 300 ad hoc adjustments are made to the source taxonomies before they are aligned to the workspace.

\section{Candidate identification}

Given a source node, the alignment procedure begins by finding the nodes in the workspace that it could possibly align with. These workspace nodes are called candidates. The candidates are simply the nodes that have a name-string (either primary or synonym) that exactly matches any name-string (primary or synonym) of the source node. (See under Future Work regarding other ways this might be done.)

(This is the ordinary language use of the word 'candidate', unrelated to 'candidatus' as used in prokaryote taxonomy.)

Example: GBIF Nakazawaea pomicola has NCBI Candida pomiphila as a candidate by way of an NCBI record that lists Nakazawaea pomicola as a synonym of Candida pomiphila.

It follows that if the workspace has multiple nodes with the same name-string (homonym name-strings), all of these nodes will become candidates for every source node that also has that name-string.

\section{Candidate selection}

The purpose of the alignment phase is to choose a single correct candidate for each source node, or to reject all candidates if none is correct. For over $97 \%$ of source nodes, there are no candidates or only one candidate, and selection is fairly simple, but the remaining nodes require special treatment.

Example: There are two nodes named Aporia lemoulti with author Bernardi in the GBIF backbone taxonomy; one is in plants and the other is in insects. The plant node is an 
erroneous duplication (recently corrected), but the automated system has to be able to cope with this situation because the sources have many errors and it is not feasible to manually correct all of them. When IRMNG is aligned, it is necessary to choose the right candidate for the node with name-string Aporia lemoulti. Consequences of incorrect placement might include putting siblings of IRMNG Aporia lemoulti in the wrong kingdom as well.

Example: Fritillaria messanensis in WoRMS must not map to Fritillaria messanensis in NCBI Taxonomy because the taxon in WoRMS is an animal (tunicate) while the taxon in $\mathrm{NCBI}$ is a flowering plant. This is a case where there is a unique candidate, but it is wrong.

Similarly, Aporia sordida is a plant in GBIF, but an insect in IRMNG.

\section{Alignment heuristics}

Once we have a list of candidates, we apply a set of heuristics in an attempt to find a single candidate, and thereby align a source node $\mathrm{n}$ ' with a workspace node $\mathrm{n}$. The heuristics are as follows, presented in the order that we apply them in the alignment process:

1. Separation: If $\mathrm{n}$ and $\mathrm{n}$ are contained in "obviously different" major groups such as animals and plants, do not align n' to n. Two major groups (or "separation taxa") are "obviously different" if they are disjoint as determined by the separation taxonomy.

Examples: (1) the Aporia cases above; (2) NCBI says $\mathrm{n}=$ Pteridium is a land plant, WoRMS says $\mathrm{n}^{\prime}=$ Pteridium is a rhodophyte, and the separation taxonomy says land plants and rhodophytes are disjoint, so $\mathrm{n}$ and $\mathrm{n}$ ' are different taxa.

2. Disparate ranks: Prohibit alignment where $\mathrm{n}$ and $\mathrm{n}$ ' have "obviously incompatible" (disparate) ranks. A rank is "obviously incompatible" with another if one is genus or a rank inferior to genus (species, etc.) and the other is family or a rank superior to family (order, etc.).

Examples: (1) IRMNG Pulicomorpha, a genus, matches NCBI Pulicomorpha, a genus, not GBIF Pulicomorpha, a suborder. Note that both candidates are insects. (2) For genus Ascophora in GBIF (which is in Platyhelminthes), candidate Ascophora from WoRMS, a genus, is preferred to candidate Ascophora from NCBI, an infraorder.

3. Lineage: Prefer to align species or genus $n$ to $n$ if they have common lineage. For example, if $n$ ' is a species, prefer candidates $n$ where the name-string of the familyrank ancestor node of $\mathrm{n}$ ' is the same as the name-string of the family-rank ancestor node of $n$.

Example: Source node Plasmodiophora diplantherae from Index Fungorum, in Protozoa, has one workspace candidate derived from NCBI and another from WoRMS. Because the source node and the NCBI candidate both claim to be in a 
taxon with name 'Phytomyxea', while the WoRMS candidate has no near lineage in common, the NCBI candidate is chosen.

The details are complicated because (a) every pair of nodes have at least some of their lineage in common, and (b) genus names do not provide any information when comparing species nodes with the same name-string, so for species we can't just look at the parent taxon. The exact rule used is the following: Define the 'quasiparent name' of $n, q(n)$, to be the name-string of the nearest ancestor of $n$ whose name-string is not a prefix of n's name-string. (For example, the quasiparent of a species would typically be a family.) If $q(n)$ is the name-string of an ancestor of $n^{\prime}$, or $q\left(n^{\prime}\right)$ is the name-string of an ancestor of $n$, then prefer $n$ to candidates that lack these properties.

4. Overlap: Prefer to align $n$ ' to $n$ if they are higher level groupings that overlap. Stated a bit more carefully: Prefern' if $n$ ' has a descendant aligned to a descendant of $n$.

Example: Source node Peranema from GBIF has two candidates from NCBI. One candidate shares descendant Peranema cryptocercum with the source taxon, while the other shares no descendants with the source taxon. The source is therefore aligned to the one with the shared descendant.

5. Proximity: Require a candidate $\mathrm{n}$ to have the property that the smallest separation taxon containing the source node $\mathrm{n}^{\prime}$ is also the smallest separation taxon containing n.

Example: Source node Heterocheilidae in IRMNG (a nematode family) has Metazoa as its smallest separation ancestor. Both workspace candidates are in families in Metazoa, but we choose the one whose smallest separation ancestor is Metazoa, not the one whose smallest separation ancestor is Diptera. As it happens, that choice is a nematode family, not a fly family.

6. Same name-string: Prefer candidates whose primary name-string is the same as the primary name-string of $n$ '.

Example: For source node n' = GBIF Zabelia tyaihyoni, candidate Zabelia tyaihyoni from NCBI is preferred to candidate Zabelia mosanensis, also from NCBI. NCBI $Z$. mosanensis is a candidate for $n$ ' because GBIF declares that $Z$. mosanensis is a synonym for GBIF Z. tyaihyoni.

\section{Control flow for applying heuristics}

Each heuristic, when presented with a source node and a candidate (workspace node), answers 'yes', 'no', or 'no information'. 'Yes' means that according to the rule, the two nodes refer to the same taxon, 'no' means they refer to different taxa, and 'no information' means that this rule provides no information as to whether the nodes refer to the same taxon. 
The answers are assigned numeric scores of 1 for yes, 0 for no information, and -1 for no. A candidate that a heuristic gives a no is eliminated, one that is unique in getting a yes is selected, and if there are no yeses or no unique yes, more heuristics are consulted.

The heuristics are applied in the order in which they are listed above. The outcome is sensitive to the ordering. The ordering is forced to some extent by internal logic, but overall the ordering was determined by trial and error.

If there is a single candidate that is not rejected by any heuristic, it is aligned to that candidate.

More specifically, the method for applying the heuristics is as follows:

1. Start with a source node $\mathrm{N}$ and its set $\mathrm{C}$ of workspace node candidates $\mathrm{C} 1 \ldots \mathrm{Cn}$.

2. For each heuristic $\mathrm{H}$ as listed above:

1. For each candidate $\mathrm{Ci}$ currently in $\mathrm{C}$, use $\mathrm{H}$ to obtain the score $\mathrm{H}(\mathrm{N}, \mathrm{Ci})$

2. Let $Z=$ the highest score from among the scores $H(N, C i)$

3. If $Z<0$, we are done - no candidate is suitable

4. Let $C^{\prime}=$ those members of $C$ that have score $Z$

5. If $Z>0$ and $C^{\prime}$ contains only one candidate, we are done (match is that candidate)

6. Otherwise, replace $C$ with $C^{\prime}$ and proceed to the next heuristic

3. If $C$ is singleton after all heuristics are exhausted, its member is taken to be the correct match.

4. Otherwise, the source node does not match unambiguously, and alignment fails.

\section{Failure to choose}

If the alignment process ends with multiple candidates, there is an unresolvable ambiguity. If the ambiguous source node has no children, it is dropped, which is OK because it probably corresponds to one of the existing candidates and therefore would make no new contribution. If the ambiguous source node has children, it is treated as unaligned and therefore new, increasing the number of workspace nodes having that name-string. This could easily be wrong because it is so unlikely that the source node really represents a distinct taxon. Usually, the subsequent merge phase determines that the grouping is not needed because it inconsistent or can be 'absorbed', and it is dropped. If it is not dropped, then there is a troublesome situation that calls for manual review.

As an example of an unaligned tip, consider GBIF Katoella pulchra. The candidates are NCBI Davallodes pulchra and Davallodes yunnanensis. (There is no Katoella pulchra in the workspace at the time of alignment. The two candidates come from synonymies with Katoella pulchra declared by GBIF.) Neither candidate is preferable to the other based on the information available to the method, so Katoella pulchra is left unaligned and is omitted from the assembly. 


\section{Redescriptions}

The scientific purpose of taxonomies is to understand taxa as historical and predictive biological abstractions. We only understand the identity of a taxon, that is, its membership or 'circumscription', through a description. For each taxon record in a taxonomy, there is a described taxon, but finding that description can be very challenging and is not this time possible at scale (although see Page 2013, Miller et al. 2015). Tragically, taxonomists frequently use the same name-string with the same authority information (author, year) to refer to different taxa. The taxa all have the same type specimen or species, thanks to general adherence to nomenclatural rules, but because the name-string + authority is associated with multiple descriptions, the taxa differ in circumscription.

Example: NCBI treats Tragopogon minor Mill. and Tragopogon dubius Scop. as separate species, while GBIF has Tragopogon minor Mill. a synonym for Tragopogon dubius Scop. The two taxonomies therefore use the name Tragopogon dubius with author Scop. to refer to different taxa, one subsuming minor and the other not. A phylogenetic OTU labeled Tragopogon dubius might be better related to OTT's Tragopogon minor record than to OTT's Tragopogon dubius record, if the specimen corresponding to the OTU were a minor but was identified by consulting a description consistent with the GBIF dubius taxon.

The large scale taxonomic sources we use provide limited help in sorting out the connection from taxon record to description. This represents a vast lost opportunity. Some halfway measures are possible. The example above was found by looking at synonyms of same-named taxa in different sources: if a name $X$ is a synonym of name $Y$ in one source, but $X$ and $Y$ have separate taxon records in another, then we can infer that the sense of name $Y$ differs between the sources.

Resolving name usages to descriptions is not a problem we're in a position to solve. And even if it were solved for the taxonomy, the phylogenetic tree files that are the input to Open Tree rarely provide the information that would help a curator choose the right taxon (record). We have made a deliberate pragmatic choice to ignore this problem, and to assume congruence of taxa in situations when name and hierarchical context agree, admitting that this practice can lead to errors in both taxonomic assembly and in phylogenetic synthesis.

\section{Merging unaligned source nodes}

After the alignment phase, we are left with the set of source nodes that could not be aligned to the workspace. The next step is to determine if and how these (potentially new) nodes can be merged into the workspace.

The combined taxonomy ( $\mathrm{U}$, above) is constructed by adding copies of unaligned nodes from the source taxonomy $S^{\prime}$ one at a time to the workspace, which initially contains a copy of $S$. Nodes of $S^{\prime}$ therefore correspond to workspace nodes in either of two ways: by mapping to a copy of an S-node (via the S'-S alignment), or by mapping to a copy of an S'node (when there is no $S^{\prime}-S$ alignment for the $S^{\prime}-$ node). 
As described above, each copied S'-node is part of either a graft or an insertion. A graft or insertion rooted at $r$ ' is attached to the workspace as a child of the nearest common ancestor node of $r$ "s siblings' images. A graft is flagged incertae sedis if that NCA is a node other than the parent of the sibling images. By construction, insertions never have this property, so an insertion is never flagged incertae sedis.

The following schematic examples illustrate each of the cases that come up while merging taxonomies. Taxonomy fragments are written in Newick notation (Olsen 1990). Fig. 3 illustrates each of these six cases.

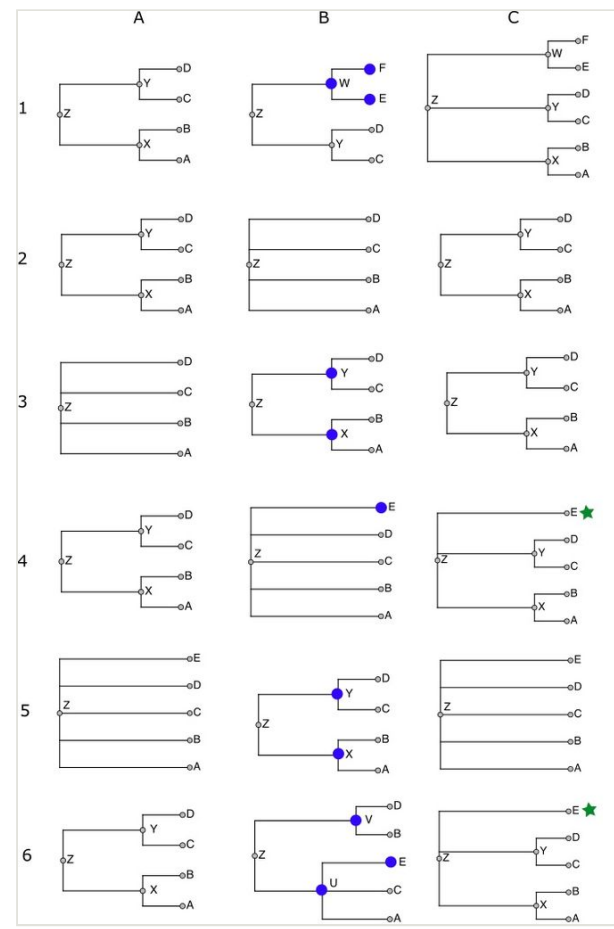

Figure 3. doi

Merging taxonomies: Examples of outcomes when merging nodes from a source taxonomy into the workspace taxonomy. Each row (1-6) corresponds to one of the six examples described in the text. Column $A$ is the current workspace taxonomy, column B is the source taxonomy being merged, and column $\mathrm{C}$ is the resulting workspace taxonomy. Nodes in $\mathrm{B}$ marked with a large blue circle are those that cannot be aligned to the workspace. Nodes in $\mathrm{C}$ marked with a green star are those flagged as incertae sedis in the final taxonomy.

Case 1: $((a, b) x,(c, d) y) z+((c, d) y,(e, f) w) z=((a, b) x,(c, d) y,(e, f) w) z$

This is a simple graft. The taxon $w$ does not occur in the workspace, so it and its children are copied. The workspace copy of $w$ is attached as a sibling of its siblings' images: its sibling is $y$ in $S^{\prime}$, which is aligned to $y$ in the workspace, so the copy becomes a child of $y$ 's parent, or $z$. 
Case 2: $((a, b) x,(c, d) y) z+(a, b, c, d) z=((a, b) x,(c, d) y) z$

No nodes are copied from S' to the workspace because every node in $S^{\prime}$ is aligned to some node in $\mathrm{S}$ - there are no nodes that could be copied.

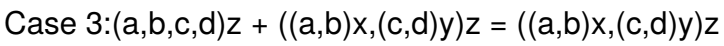

Supposing $\mathrm{x}$ and $\mathrm{y}$ are unaligned, then $\mathrm{x}$ and $\mathrm{y}$ from $\mathrm{S}^{\prime}$ insert into the classification of $z$. The workspace gets copies of these two $S^{\prime}$-nodes.

Example: superfamily Chitonoidea, which is in WoRMS (S') but not in NCBI Taxonomy (S), inserts into NCBI Taxonomy. Its parent is suborder Chitonina, which is in NCBI (i.e. aligned to the workspace), and its children are six families that are all in NCBI (aligned).

Case 4: $((a, b) x,(c, d) y) z+(a, b, c, d, e) z=((a, b) x,(c, d) y, ? e) z$

In this situation, we don't know where to put the unaligned taxon e from $S^{\prime}$ : in $x$, in $y$, or in $z$ (sibling to $x$ and $y$ ). The solution used here is to add $e$ to $z$ and mark it as incertae sedis (indicated above by the question mark).

For example, family Melyridae from GBIF has five genera, of which two (Trichoceble , Danacaea) are not found in the workspace, and the other three do not all have the same parent after alignment - they are in three different subfamilies. Trichoceble and Danacaea are made to be incertae sedis children of Melyridae, because there is no telling which NCBI subfamily they are supposed to go in.

Case 5: $(a, b, c, d, e) z+((a, b) x,(c, d) y) z=(a, b, c, d, e) z$

We don't want to lose the fact from the higher priority taxonomy $S$ that $\mathrm{e}$ is a proper child of $z$ (i.e. not incertae sedis), so we discard nodes $x$ and $y$, ignoring what would otherwise have been an insertion.

So that we have a term for this situation, say that $\mathrm{x}$ is absorbed into $\mathrm{z}$.

Case 6: $((a, b) x,(c, d) y) z+((a, c) p,(b, d, e) q) z=((a, b) x,(c, d) y, ? e) z$

If the source has a hierarchy that is incompatible with the one in the workspace, the conflicting source nodes are ignored, and any unaligned nodes (e) become incertae sedis nodes under an ancestor containing the incompatible node's children.

For example, when WoRMS is merged, the workspace has, from NCBI,

((Archaeognatha)Monocondylia,(Pterygota,Zygentoma)Dicondylia)Insecta

and the classification given by WoRMS is

((Archaeognatha, Thysanura=Zygentoma)Apteryogota,Pterygota)Insecta 
That is, NCBI groups Thysanura (Zygentoma) with Pterygota, while WoRMS groups it with Archaeognatha. The WoRMS hierarchy is ignored in favor of the higher priority NCBI hierarchy. If Insecta in WoRMS had had an unaligned third child, it would have ended up incertae sedis in Insecta.

The test for compatibility is very simple: a source node is incompatible with the workspace if the nodes that its aligned children align with do not all have the same parent.

\section{Merging node data}

A new workspace node gets its data from the unaligned source node, including its namestrings and primary name-string designation. The merge phase records, in each workspace node, a reference to the unaligned source taxonomy node from which it was copied, allowing creation of an actionable hyperlink. This provenance tracking has proven to be quite valuable.

For aligned nodes, source name-strings that were not already present are added to the set of workspace node name-strings (also tracking the relevant source node for each). References to aligned source nodes are recorded in workspace nodes at this point, so that at the end of assembly, each workspace node records the initial source node that contributed it as well as all the source nodes that aligned to it.

\section{Final patches}

After all source taxonomies are aligned and merged, we apply general ad hoc additions and patches to the workspace, in a manner similar to that employed with the source taxonomies. Patches are represented in three formats. An early patch system used handwritten tabular files, additions via the user interface use a machine-processed JSON format, and most other patches are written as simple Python statements. There are 106 additions in JSON form, 97 additions and patches in tabular form, and approximately 121 in Python form.

\section{Assigning identifiers}

The final step is to assign unique, stable identifiers to nodes so that external links to OTT nodes will continue to function correctly after the previous OTT version is replaced by the new one.

Identifier assignment is done by aligning the previous version of OTT to the new version. As with the other alignments, there are scripted ad hoc adjustments to correct for some errors that would otherwise be made by automated assignment. For this alignment, the set of heuristics is extended by adding rules that prefer candidates that have the same source taxonomy node id as the previous version node being aligned. After transferring identifiers of aligned nodes, any remaining workspace nodes are given newly 'minted' identifiers. 
The alignment is computed only for the purpose of assigning identifiers; the previous OTT version is not merged into the workspace. An identifier can only persist from one OTT version to the next if it continues to occur in some source taxonomy.

\section{Results}

The assembly method described above yields the reference taxonomy that is used by the Open Tree of Life project. The taxonomy itself, the details of how the assembly method unrolls to generate the taxonomy, and the degree to which the taxonomy meets the goals set out for it are all of interest in assessing how, and how well, the method works. We will address each of these three aspects of the method in turn.

\section{Summary of Open Tree Taxonomy}

The methods and results presented here are for version 3.0 of the Open Tree Taxonomy (which follows five previous releases using the automated assembly method). The taxonomy contains $3,594,550$ total taxa; 3,272,177 tips; and 277,365 internal nodes. $2,335,412$ of the nodes have a Linnean binomial of the form Genus epithet. There are $1,842,403$ synonym records and 9,089 name-strings that are primary for more than one nodes. A longer list of metrics is in Table 2.

Table 2.

Summary of Open Tree Taxonomy 3.

\begin{tabular}{|l|l|}
\hline Number nodes & Property \\
\hline 3594550 & Total taxon records (nodes) \\
\hline 1842403 & Synonym records \\
\hline 277365 & Internal (non-tip) nodes \\
\hline 3272177 & Tips. \\
\hline 3116485 & Rank of 'species' \\
\hline 70886 & Below the rank of species (e.g. subspecies, variety) \\
\hline 67070 & Above the rank of species that subtend no node of rank species \\
\hline 2335412 & Name-string has the form of a Linnaean binomial Genus epithet \\
\hline 9089 & Homonym name-strings \\
\hline 2867 & Homonym name-strings where the nodes have species rank \\
\hline 6110 & Homonym name-strings where the nodes have genus rank \\
\hline 38 & Maximum nesting depth of any node in the taxonomy \\
\hline 53287 & Maximum number of children for any node in the taxonomy \\
\hline
\end{tabular}




\begin{tabular}{|l|l|}
\hline 12.96 & Branching factor (average number of children per internal node) \\
\hline 45008 & Source taxa that were absorbed into a larger taxon \\
\hline 317624 & Marked incertae sedis or equivalent \\
\hline 252600 & Annotated as being for an extinct taxon \\
\hline
\end{tabular}

The number of species level homonym name-strings (2867) is surprisingly high. While a small number of these are legitimate, e.g. Scoparia dulci which is used in practice for both a plant and an insect, most of them result from errors in sources. Some originate in a single source, but most seem to be between nodes contributed by multiple sources. We researched ten cases chosen at random, and in every one, two sources disagreed on placement in separation taxa, and only one source is correct. E.g. Callirhynchius exquisitus is in beetles in one source and in decapods in another, so the Separation heuristic prevents alignment, and the workspace ends up with two nodes. But the decapod placement is incorrect, and there is really only one species. (Amazingly, every one of the ten samples was later corrected in the source database!)

\section{Results of assembly procedure}

As OTT is assembled, the alignment procedure processes every source node, either choosing an alignment target for it in the workspace based on the results of the heuristics, or leaving it unaligned. Fig. 4 illustrates the action of the alignment phase. The presence of a single candidate node does not automatically align the two nodes - we still apply the heuristics to ensure a match (and occasionally reject the single candidate).

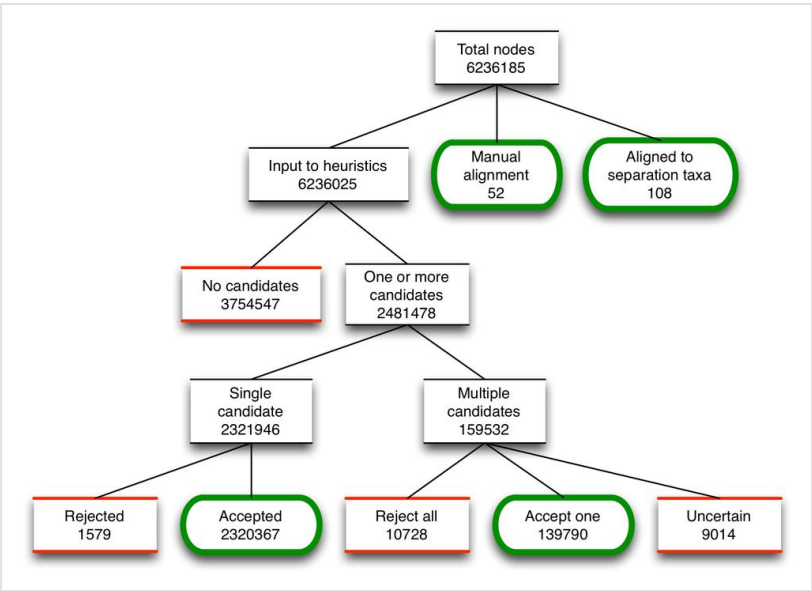

Figure 4. doi

Fate of nodes as they move through the alignment procedure. Green, rounded boxes are endpoints that result in aligned nodes, while red, sqaure boxes are endpoints that result in unaligned nodes. 
We counted the frequency of success for each heuristic, i.e. the number of times that a particular heuristic was the one that accepted the winning candidate from among two or more candidates. Table 3 shows these results. Separation (do not align taxa in disjoint separation taxa; used first), Lineage (align taxa with shared lineage; used midway through) and Same-name-string (prefer candidates who primary name-string matches; used last) were by far the most frequent.

Table 3.

Frequency of success of alignment heuristics. In cases where there were multiple candidate nodes, this table lists the number of times that a particular heuristic was the one to select a single candidate. Heuristics are listed in the order in which they are applied. Success of an ealier heuristics means that a later heuristic is not used for a given node.

\begin{tabular}{|l|l|}
\hline Alignment heuristic & Number nodes \\
\hline Separation & 22126 \\
\hline Disparate ranks & 154 \\
\hline Lineage & 25688 \\
\hline Overlap & 7438 \\
\hline Proximity & 228 \\
\hline Same name-string & 84211 \\
\hline
\end{tabular}

After assembly, the next step in the method is to merge the unaligned nodes into the workspace taxonomy. Of the 3,780,949 unaligned nodes, the vast majority (99\%) are grafted into the workspace. The remaining nodes $(<1 \%)$ are either insertions, absorptions or remain unmerged due to ambiguities.

We also examined the fate of nodes from each of the input taxonomies, and Table 4 provides these results. The results are dependent on the order in which sources are added to the workspace. Overall, the number of conflicts is relatively low $(<1 \%)$.

Table 4.

Fate of source nodes from each of the input taxonomies. Unaligned nodes are either copied into the workspace or absorbed. Aligned nodes are added to the workspace through grafting or insertion.

\begin{tabular}{|l|l|l|l|l|l|}
\hline Source & Total & Copied & Aligned & Absorbed & Conflict \\
\hline separation & 30 & 30 & 0 & 0 & 0 \\
\hline SILVA & 74400 & 74395 & 5 & 0 & 0 \\
\hline Hibbett 2007 & 227 & 226 & 1 & 0 & 0 \\
\hline Index Fungorum & 276262 & 276048 & 188 & 25 & 1 \\
\hline WoRMS & 327570 & 269029 & 57026 & 1283 & 232 \\
\hline Schäferhoff 2010 & 119 & 118 & 1 & 0 & 0 \\
\hline
\end{tabular}




\begin{tabular}{|l|l|l|l|l|l|}
\hline NCBI & 1320665 & 1198221 & 119532 & 2441 & 471 \\
\hline GBIF & 2451566 & 1640700 & 808757 & 1963 & 146 \\
\hline IRMG & 1561123 & 90746 & 1466929 & 3128 & 320 \\
\hline curated & 29 & 29 & 0 & 0 & 0 \\
\hline total & 6011991 & 3549542 & 2452439 & 8840 & 1170 \\
\hline
\end{tabular}

\section{Evaluating the taxonomy relative to requirements}

The introduction sets out requirements for an Open Tree taxonomy. How well are these requirements met?

\section{OTU coverage}

We set out to cover the OTUs in the Open Tree corpus of phylogenetic trees. The corpus contains published studies (each study with one or more phylogenetic trees) that are manually uploaded and annotated by Open Tree curators. The user interface contains tools that help curators map the OTUs in a study to taxa in OTT. Of the 3,242 studies in the Open Tree database, 2,871 have at least $50 \%$ of OTUs mapped to OTT. (A lower overall mapping rate usually indicates incomplete curation, not an inability to map to OTT.) These 2,871 studies contain 538,728 OTUs, and curators have mapped 514,346 to OTT taxa, or $95.5 \%$.

To assess the reason for the remaining $4.5 \%$ of OTUs being unmapped, we investigated a random sample of ten OTUs. In three cases, the label was a genus name in OTT followed by "sp" (e.g. "Euglena sp"), suggesting the curator's unwillingness to take the genus as the correct mapping for the OTU. In the remaining seven cases, the taxon was already in OTT, and additional curator effort would have found it. Two of these were misspellings in the phylogenetic tree file; one was present under a slightly different name-string (subspecies in OTT, species in study, the study reflecting a very recent reclassification); and in the remaining four cases, either the taxon was added to OTT after the study was curated, or the curation task was left incomplete. None in the sample reflected a coverage gap.

Of the 194,100 OTT records that are the targets of OTUs, $188,581(97.2 \%)$ are represented in NCBI Taxonomy. If the Open Tree project had simply adopted NCBI Taxonomy instead of OTT, it would have met its OTU coverage requirement (but not the taxonomic coverage requirement). By comparison, GBIF covers $87.6 \%$, and IRMNG covers $62.8 \%$. The high coverage by $\mathrm{NCBI}$ reflects a preference among Open Tree curators for studies that use molecular phylogenetic evidence over those that don't.

\section{Phylogenetically informed classification}

Assessing whether OTT is more 'phylogenetically informed' than it otherwise might be is difficult. The phylogenetic quality of the taxonomy is determined by the taxonomic sources 
and their priority order. We have relied on the project's curators, who have a strong phylogenetic interest, to provide guidance on both. Following are examples of curator decision-making:

- For microbes, SILVA is considered more phylogenetically sound than NCBI taxonomy, because the SILVA taxonomy is based on a recent comprehensive phylogenetic analysis.

- Priority of NCBI Taxonomy over the GBIF backbone is suggested by NCBI's apparent interest in phylogeny, reflected in NCBI Taxonomy's much higher resolution, its inclusion of phylogenetically important non-Linnaean groups such as Eukaryota, and by its avoidance of known paraphyletic groupings such as Protozoa.

- The Hibbett 2007 upper fungal taxonomy reflects, by construction, results from the most recent phylogenetic studies of Fungi.

Ideally we would have a measure of 'phylogenetically informed' that we could use to compare OTT to other taxonomies, to test alternative constructions of OTT, and to check the forward progress of OTT. It is not clear what one would use as a standard against which to judge. The Open Tree project's synthetic tree of life is a candidate, but is not without issues (such as its own possible errors, and the fact that OTT is itself use in construction the synthetic tree). Ensuring that comparisons are meaningful, and comparable with one another, would be a technical challenge.

\section{Taxonomic coverage}

OTT has 2.3M binomials (presumptive valid species names), vs. 1.6M for Catalogue of Life (CoL) or 2.2M for GBIF. Since the GBIF source we used includes the Catalogue of Life, OTT includes all species in CoL. It is not clear whether the differences between OTT and GBIF, and between GBIF and CoL, are due to mostly to inclusion of additional accepted names, versus names that are not currently accepted (synonym, invalid, and so on); this is not a question we investigated.

This level of coverage would seem to meet Open Tree's taxonomic coverage requirement as well as any other available taxonomic source.

\section{Ongoing update}

We aimed for a procedure that would allow simple re-building from sources, and also easy incorporation of new versions of sources. Re-building OTT version 3.0 from sources requires 17 minutes of real time. Our process currently runs on a machine with 16GB of memory; $8 \mathrm{~GB}$ is not sufficient.

In the upgrade from 2.10 to 3.0, we added new versions of both NCBI and GBIF. NCBI updates frequently, so changes tend to be manageable and incorporating the new version was simple. In contrast, the version from GBIF represented both a major change in their 
taxonomy synthesis method. Many taxa disappeared, requiring changes to our ad hoc patches during the normalization stage. In addition, the new version of GBIF used a different taxonomy file format, which requires extensive changes to our import code (most notably, handling taxon name-strings that now included authority information).

We estimate the update from OTT 2.10 to OTT 3.0 required approximately three days of development time related to source taxonomy changes. This was greater than previous updates due to the changes required to handle the major changes in GBIF content and format.

\section{Open data}

All sources are available on the Internet without access controls. We considered legal restrictions that might apply to OTT's particular use of the source materials, and are satisfied that they do not. In particular, copyright does not apply because the taxonomy is not creative expression in the sense of copyright law (Patterson et al. 2014). We have marked OTT with a Creative Commons CC0 version 1.0 waiver (Steuer 2007).

In any kind of compilation, sources need to be attributed regardless of legal considerations. For OTT sources this is accomplished in two ways. First, every record (node) has a hyperlink to the source taxonomy record from which it was originally copied, as well as one to each of the source records that aligned to it. Second, the release notes list the sources with version information and references to relevant publications and web sites.

\section{Discussion}

The primary actionable information in the source taxonomies consists of name-strings, and therefore the core of our method is a set of heuristics that can handle the common problems encountered when trying to merge hierarchies of name-strings. These problems include expected taxonomic issues such as synonyms, homonyms, and differences in placement and membership between sources. They also include errors such as duplications, spelling mistakes, and misplaced taxa. The problem cases add up to over 100,000 difficult alignments when the total number of source records measures over 6 million.

Ultimately there is no fully automated and foolproof test to determine whether two nodes can be aligned - whether node $A$ and node $B$, from different source taxonomies, are about the same taxon. The information to do this is in the literature and in databases on the Internet, but often it is (understandably) missing from the source taxonomies.

It is not feasible to investigate such problems individually, so the taxonomy assembly methods identify and handle thousands of 'special cases' in an automated way. We currently use only name-strings, rudimentary classification information, and (minimally) ranks to guide assembly. We note the large role that our hand-curated "separation taxonomy" played in the alignment phase. This is a set of taxa that are consistent across 
the various sources, and allow us to make the (seemingly obvious) determination "these two taxa are in completely separate groups, so do not align them".

\section{Community curation}

We have also developed a system for curators to directly add new taxon records to the taxonomy from published phylogenies, which often contain newly described species that are not yet present in any source taxonomy. These taxon records include provenance information, including references explaining the taxon, and the identity of the curator. We expose this provenance information through the web site and the taxonomy API.

We also provide a feedback mechanism on the synthetic tree browser, and find that most of the comments left are about presence, absence, choice, and spelling of labels, rather than the topology of the synthetic tree. These are issues that are addressed by improvements to the taxonomy. Expanding this feature to capture this feedback in a more structured, and therefore machine-readable, format would allow users to directly contribute taxonomic patches to the system.

\section{Comparison to other taxonomies}

Given the unique goals of the Open Tree Taxonomy in comparison to most other taxonomy projects, it is difficult to compare OTT to other taxonomies in a meaningful way. The Open Tree Taxonomy is technically most similar to the GBIF taxonomy, in the sense that each is a synthesis of existing, overlapping taxonomies rather than a curated taxonomic database or one based on grafting. The GBIF method is yet unpublished (for basic information on the GBIF backbone see Döring 2016a, Döring 2016b). Once the GBIF method has been formally described, it will be useful to compare the two approaches and identify common and unique techniques for automated, scalable name-string matching and hierarchy merging.

\section{Potential improvements and future work}

The development of the assembly software has been driven by the needs of the Open Tree project, not by any concerted effort to create a widely applicable or theoretically principled tool. A system like this is never finished, and this one is in its infancy. There are endless opportunities for bringing additional techniques, methods, data, and code libraries to bear, and we have faced difficult choices in deciding where to put our effort. Following are some of the directions for development that could have the highest impact.

- It is likely that alignment and merge could be improved by making better use of species proximity implied by the shape of the classification, and decreasing its reliance on the names of internal nodes. Better use of proximity might permit separation and identification of tips without use of a separation taxonomy, removing the need for the manual work of maintaining the separation taxonomy and the adjustment directives needed to align source taxonomies to it. An example is 
Conolophus, where two genus-level nodes in the same separation taxon are mistakenly combined. How to accomplish this is not obvious, but it is not obviously impossible.

- The alignment method should be extended to make use of authority information, when it is available. If name-strings match, or even if just species epithets match, then matching authority information is good evidence that the same taxon is meant. The form of authority information varies between sources, but could be normalized using the Global Names Parser (Patterson et al. 2016).

- Name-strings could also be analyzed to detect partial matches, e.g. matching on species epithets even when the genus disagrees, and spelling and gender variant recognition. Doing so would eliminate thousands of duplications. Other work on name matching, such as the Global Names Resolver (Patterson et al. 2016), goes far beyond what is done for OTT and these techniques should be used.

- The redescription problem described above should be addressed to the extent possible.

- $\quad$ Our handling of duplicate records in source taxonomies is incomplete and needs to be fixed. If two source nodes can be aligned to the same workspace node, then the duplication will not affect the workspace. But if there is no workspace node to align them to, the duplication persists in the workspace after the source taxonomy has been merged. There is special case logic when taxonomies are imported to fold together sibling duplicates, but not alignable duplicates generally.

- An assembly run can lead to a variety of error conditions and test failures. Currently these are difficult to diagnose, mainly for lack of technology for displaying the particular pieces of the sources, workspace, and assembly history that are relevant to the error. Once this information is surfaced it is usually not too difficult to work out a fix in the form of a patch or an improvement to the program logic. A small amount of automation could speed this kind of investigation and save curator time.

- The community curation should be developed, as mentioned above. Its success would depend on allowing users to test proposed changes and diagnose and repair any problems with them.

- $\quad$ Curators frequently request new taxonomy sources. The most frequently requested are improved fish, bird, plant, and paleontological sources. Community members have also suggested the Plazi TreatmentBank (Miller et al. 2015). Again, the information is generally available, but not yet harvested. (Some frequently requested sources may only be accessed under agreement with contractual terms (variously called "terms of use" or a "data use agreement"). One of these is the IUCN Red List (International Union for Conservation of Nature and Natural Resources 2016), an important source of up-to-date information on mammal species. These sources are off limits to Open Tree due to the project's open data requirement.) 
- The presence of invalid and unaccepted names remains a significant problem. The information needed to detect them is available, and could be harvested.

- Basic usability features for application to new projects would include proper packaging of the application, and support for Darwin Core (Wieczorek et al. 2012) for both input and output.

Future work on taxonomy aggregation should attempt a more rigorous and pluralistic approach to classification (Franz et al. 2016a, Kennedy et al. 2006, Lepage et al. 2014, Franz et al. 2016b). Alignment should detect and record lumping and splitting events, and the classification conflicts detected during merge should be exposed to users. Exposing conflicts is in the interest of scientific transparency. Retaining alternative groupings could be useful in phylogenetic analysis, as a check on which of the sources agree or disagree with a given analysis. Lumping and splitting due to redescription, which lead to the same name-string (including author) referring to different taxa in different sources, could be recorded using multiple nodes qualified by description or source ('sensu'). Ideally, better handling of descriptions in aggregators ought to encourage sources to make links to primary sources more readily available for a variety of purposes.

\section{Data resources}

All source code is open source (licensed BSD 2-clause) and available on GitHub at https:// github.com/OpenTreeOfLife/reference-taxonomy. A snapshot of the code used to produce the version of OTT described here is archived at Zenodo (https://doi.org/10.5281/ zenodo.546111). All data, including Open Tree Taxonomy 3.0 and all processed source taxonomies is archived on Dryad (cannot be uploaded before paper accepted; version for review at https://github.com/OpenTreeOfLife/reference-taxonomy/tree/master/doc/method/ data-package).

\section{Conclusions}

We have presented a method for merging multiple taxonomies into a single synthetic taxonomy. The method is designed to produce a taxonomy optimized for the Open Tree of Life phylogenetic tree synthesis project. Most taxonomy projects are databases of taxonomy information that are continuously updated by curators as new information is published in the taxonomic literature. In contrast, the Open Tree Taxonomy takes several of these curated taxonomies and assembles a synthetic taxonomy de novo each time a new version of the taxonomy is needed.

We have also developed a system for curators to directly add new taxa to the taxonomy from published phylogenies. These taxon additions include provenance information, including the source of the taxon and identity of the curator. We expose this provenance information through the website and the taxonomy API. Most of the Open Tree feedback has been about taxonomy, and expanding this feature to other types of taxonomic 
information allows users to directly contribute expertise and allows projects to easily share that information.

Taxonomic information is certainly best curated at a scale smaller than "all life" by experts in a particular group. Therefore, producing comprehensive taxonomies is always a synthesis of curated taxonomies. We advocate for the type of methods being used by Open Tree and by GBIF, where synthesis of overlapping sources is done in a repeatable fashion from sources, allowing changed information in sources to be quickly included in the comprehensive taxonomy, and also allowing continuous improvement to the synthesis method. Provenance information is retained and presented as part of the synthetic taxonomy. This type of synthesis requires that source taxonomies be available online, either through APIs or by bulk download, in a format that can be easily parsed, and ideally without terms of use that prevent distribution and reuse of the resulting synthetic taxonomies.

\section{Acknowledgements}

We would like to thank Nico Franz, Mark Holder, and Alan Ruttenberg for their comments on early versions of this manuscript; Markus Döring, Tony Rees, and Paul Kirk for answering our many questions about source taxonomies; Cody Hinchliff for writing an early version of the assembly code and establishing the general approach to taxonomy combination; and Yan Wong and other users of the Open Tree system provided many helpful comments on the taxonomy. The reviewers' comments were very thoughtful and thorough and led to many important improvements to the presentation.

\section{Funding program}

NSF AVAToL (Assembling Visualizing, and Analyzing the Tree of Life) DEB-1208809

\section{Grant title}

Automated and community-driven synthesis of the tree of life

\section{Hosting institution}

Duke University 


\section{Author contributions}

JAR designed and implemented the taxonomy assembly system; KAC and JAR wrote the paper.

\section{Conflicts of interest}

The authors declare no conflicts of interest.

\section{References}

- Döring M (2016a) Updating the GBIF Backbone. GBIF Developer Blog URL: http:// gbif.blogspot.com/2016/04/updating-gbif-backbone.html

- $\quad$ Döring M (2016b) GBIF Backbone - August 2016 Update. GBIF Developer Blog URL: http://gbif.blogspot.com/2016/08/gbif-backbone-august-2016-update.html

- Edwards J (2004) Research and societal benefits of the global biodiversity information facility. BioScience 54 (6): 485. https://doi.org/10.1641/0006-3568(2004)054 [0486:RASBOT]2.0.CO;2

- $\quad$ Federhen S (2011) The NCBI Taxonomy database. Nucleic Acids Research 40: D136-D14. https://doi.org/10.1093/nar/gkr1178

- Franz N, Gilbert E, Ludäscher B, Weakley A (2016a) Controlling the taxonomic variable: Taxonomic concept resolution for a southeastern United States herbarium portal. Research Ideas and Outcomes 2: e10610. https://doi.org/10.3897/rio.2.e10610

- $\quad$ Franz N, Pier N, Reeder D, Chen M, Yu S, Kianmajd P, Bowers S, Ludäscher B (2016b) Two Influential Primate Classifications Logically Aligned. Systematic Biology 65 (4): 561-582. https://doi.org/10.1093/sysbio/syw023

- Hibbett DS, Binder M, Bischoff JF, Blackwell M, al e (2007) A higher-level phylogenetic classification of the Fungi . Mycological Research 111 (5): 509-547. https:// doi.org/10.1016/i.mycres.2007.03.004

- Hinchliff C, Smith S, Allman J, Burleigh JG, Chaudhary R, Coghill L, Crandall K, Deng J, Drew B, Gazis R, Gude K, Hibbett D, Katz L, Laughinghouse HD, McTavish EJ, Midford P, Owen C, Ree R, Rees J, Soltis D, Williams T, Cranston K (2015) Synthesis of phylogeny and taxonomy into a comprehensive tree of life. Proceedings of the National Academy of Sciences 112 (41): 12764-12769. https://doi.org/10.1073/pnas.1423041112

- Index Fungorum Partnership (2014) Index Fungorum. http://www.indexfungorum.org. Accessed on: 2014-4-07.

- International Union for Conservation of Nature and Natural Resources (2016) The IUCN Red List of Threatened Species, version 2016-3. http://www.iucnredlist.org/. Accessed on: 2017-3-01.

- Kennedy J, Hyam R, Kukla R, Paterson T (2006) Standard Data Model Representation for Taxonomic Information. OMICS: A Journal of Integrative Biology 10 (2): 220-230. https://doi.org/10.1089/omi.2006.10.220 
- $\quad$ Lepage D, Vaidya G, Guralnick R (2014) Avibase - a database system for managing and organizing taxonomic concepts. ZooKeys 420: 117-135. https://doi.org/10.3897/ zookeys.420.7089

- $\quad$ Lin C, Qiao H, Wang J, Ji L, Patterson D (2016) Taxonomic Tree Tool: A tool for managing and comparing taxonomies. http://documents.tips/documents/taxonomic-treetool.html. Accessed on: 2017-4-07.

- McTavish EJ, Hinchliff C, Allman J, Brown J, Cranston K, Holder M, Rees J, Smith S (2015) Phylesystem: a git-based data store for community-curated phylogenetic estimates: Fig. 1. Bioinformatics 31 (17): 2794-2800. https://doi.org/10.1093/ bioinformatics/btv276

- $\quad$ Miller J, Agosti D, Penev L, Sautter G, Georgiev T, Catapano T, Patterson D, King D, Pereira S, Vos R, Sierra S (2015) Integrating and visualizing primary data from prospective and legacy taxonomic literature. Biodiversity Data Journal 3: e5063. https:// doi.org/10.3897/bdj.3.e5063

- $\quad$ Olsen G (1990) Interpretation of the "Newick's 8:45" tree format standard. http:// evolution.genetics.washington.edu/phylip/newick doc.html. Accessed on: 2017-3-01.

- Open Tree of Life project (2017) Open Tree of Life: Exploring the current synthetic tree. https://tree.opentreeoflife.org/. Accessed on: 2017-1-02.

- Page RD (2008) Biodiversity informatics: the challenge of linking data and the role of shared identifiers. Briefings in bioinformatics 9 (5): 345-354. https://doi.org/10.1093/bib/ bbn022

- $\quad$ Page RM (2013) BioNames: linking taxonomy, texts, and trees. PeerJ 1: e190. https:// doi.org/10.7717/peeri.190

- $\quad$ Patterson D (2014) Helping Protists to Find Their Place in a Big Data World. Acta Protozoologica 53: 115-28. https://doi.org/10.4467/16890027AP.14.011.1448

- Patterson D, Mozzherin D, Shorthouse D, Thessen A (2016) Challenges with using names to link digital biodiversity information. Biodiversity Data Journal 4: e8080. https:// doi.org/10.3897/BDJ.4.e8080

- $\quad$ Patterson DJ, Egloff W, Agosti D, Eades D, Franz N, Hagedorn G, Rees JA, Remsen DP (2014) Scientific names of organisms: attribution, rights, and licensing. BMC research notes 7: 79. https://doi.org/10.1186/1756-0500-7-79

- $\quad$ Pyle R (2016) Towards a Global Names Architecture: The future of indexing scientific names. ZooKeys 550: 261-281. https://doi.org/10.3897/zookeys.550.10009

- Quast C, Pruesse E, Yilmaz P, Gerken J, Schweer T, Yarza P, Peplies J, Glöckner F (2013) The SILVA ribosomal RNA gene database project: improved data processing and web-based tools. Nucleic Acids Research 41 (D1): D590-D596. https://doi.org/10.1093/ nar/gks1219

- Redelings B, Holder M (2017) A supertree pipeline for summarizing phylogenetic and taxonomic information for millions of species. PeerJ 5: e3058. https://doi.org/10.7717/ peerj.3058

- $\quad$ Rees T (2008) IRMNG - The Interim Register of Marine and Nonmarine Genera. Provisional Abstracts of the 2008 Annual Conference of the Taxonomic Databases Working Group. Biodiversity Information Standards (TDWG) and the Missouri Botanical Garden URL: http://www.tdwg.org/fileadmin/2008conference/documents/ 
- $\quad$ Schäferhoff B, Fleischmann A, Fischer E, Albach D, Borsch T, Heubl G, Müller K (2010) Towards resolving Lamiales relationships: insights from rapidly evolving chloroplast sequences. BMC Evolutionary Biology https://doi.org/10.1186/1471-2148-10-352

- Shipunov A (2011) The problem of hemihomonyms and the on-line hemihomonyms database (HHDB). Bionomina https://doi.org/10.11646/bionomina.4.1.3

- Species 2000 (2017) About [Catalogue of Life]. http://catalogueoflife.org/content/about. Accessed on: 2017-4-07.

- Steuer E (2007) Creative Commons launches CC+ and CCO programs. CC Newsletter (5)12. URL: https://mirrors.creativecommons.org/newsletter/ccnewsletter5.pdf\#page =12 Wieczorek J, Bloom D, Guralnick R, Blum S, Döring M, Giovanni R, Robertson T (2012) Darwin Core: An evolving community-developed biodiversity data standard. PLoS ONE 7 (1): e29715. https://doi.org/10.1371/journal.pone.0029715

- WoRMS Editorial Board (2015) World Register of Marine Species. http:// www.marinespecies.org. Accessed on: 2015-10-01. 